\title{
Inhibition of vascular adventitial remodeling by netrin-1 in diabetic rats
}

\author{
Hui-Fang Wang ${ }^{1}$, Qing-Qing Yu' ${ }^{1}$, Rui-Fang Zheng ${ }^{2}$ and Ming Xu' ${ }^{1}$ \\ 1Department of Clinical Pharmacy, School of Preclinical Medicine and Clinical Pharmacy, China Pharmaceutical University, Nanjing, Jiangsu, China \\ ${ }^{2}$ Xinjiang Key Laboratory of Uighur Medicines, Xinjiang Institute of Materia Medica, Urumqi, Xinjiang, China
}

Correspondence should be addressed to M Xu: mingxu@cpu.edu.cn

\begin{abstract}
Cardiovascular complications of type 2 diabetes mellitus (T2DM) are associated with vascular remodeling in the arteries. Perivascular sympathetic neurons release an abundance of trophic factors to regulate vascular function via a paracrine signaling. Netrin-1, a diffusible protein that can be secreted outside the cell, is one of common signals of 'conversation' between nerve and vessel. The present study investigated whether netrin-1 is a novel modulator of sympathetic neurons paracrine signaling and played a critical role in vascular adventitial remodeling under T2DM. Vascular adventitial remodeling was observed in adventitial fibroblasts (AFs) responding to netrin-1 deficiency in the supernatant from primary rat superior cervical ganglia (SCG) neurons, shown as AFs proliferation, migration, and collagen deposition. Conditioned medium from the high glucose (HG)-treated SCG neurons contributed to AFs remodeling, which was effectively alleviated by exogenous netrin-1 supplementation. Further, it was found that uncoordinated-5-B (Unc5b) was mainly expressed in AFs among netrin-1 specific receptors. Treatment of netrin-1 inhibited $\mathrm{H}_{2} \mathrm{O}_{2}$ production derived from NADPH oxidase 4 (NOX4) through the UNC5b/CAMP/PKA signal pathway in AFs remodeling. In vivo, aorta adventitial remodeling was accompanied with the downregulation of netrin-1 in the perivascular sympathetic nerve in T2DM rats. Such abnormalities were restored by netrin-1 intervention, which was associated with the inhibition of NOX4 expression in the aorta adventitia. In conclusion, netrin-1 is a novel modulator of sympathetic neurons paracrine signaling to maintain AFs function. Vascular adventitial remodeling was aggravated by sympathetic neurons paracrine signaling under hyperglycemia, which was ameliorated by netrin-1 treatment through the UNC5b/CAMP/PKA/NOX4 pathway.
\end{abstract}

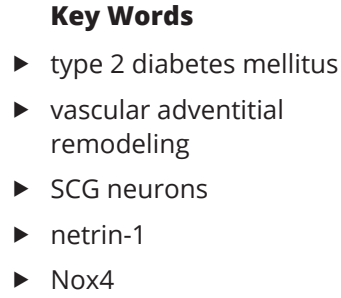

Journal of Endocrinology (2020) 244, 445-458

\section{Introduction}

Cardiovascular complications of diabetes are associated with vascular remodeling in large and small arteries (Salum Erik et al. 2012). In recent years, compelling evidences have suggested that the adventitia is a crucial regulator of vascular structure and function from the 'outside-in' (Jean-Baptiste et al. 2007). Adventitial remodeling in systemic vasculature is characterized by AFs proliferation, which precedes and exceeds endothelial and smooth muscle cell proliferation. It is believed that AFs are often the first vascular cells to be activated and reprogrammed under pathological condition (Jean-Baptiste et al. 2007, Stenmark et al. 2013). Therefore, the critical mechanisms about vascular adventitial remodeling have been paid more and more attention. 
The sympathetic nervous system, a subdivision of the autonomic nervous system, plays an essential role in the regulation of vascular contractility and structure. Fibroblast distribution roughly parallels sympathetic nerve distribution in the vessels and perivascular sympathetic axons surround the vessels in lace-like networks. The sympathetic nerve fibers are confined to the vascular media-adventitia border above the medial smooth muscle cell layer (Soares-Da-Silva \& Azevedo 1985, Kacem \& Sercombe 2006, Burnstock 2008). The sympathetic innervation not only influences AFs phenotype and growth, but also prevents the migration of AFs during post-natal development (Kacem \& Sercombe 2008). On the other hand, the alterations in vascular sympathetic innervation have been implicated in the development of cardiovascular disease (Zhang \& Faber 2001, Sheng \& Zhu 2018). The perivascular sympathetic nerves release an abundance of trophic factors to act on various receptors to regulate vascular function by a paracrine signaling. However, the communication signal between sympathetic neurons and AFs has not been fully established due to some controversial data (Burnstock 2008, Sheng \& Zhu 2018).

Netrin-1 is originally identified as a midline-derived chemoattractant that guides axons to the midline during development. Meanwhile, netrin-1 is a diffusible protein that can be secreted in the extracellular environment as one of the common signals of 'conversation' between nerves and vessels (Kennedy et al. 1994, Carmeliet \& Tessier-Lavigne 2005). Recently, more observations found that netrin-1 was expressed in both the adult central and peripheral nervous system and was secreted by oligodendrocytes, myelinating cells, and schwann cells (Madison et al. 2000, Rajasekharan \& Kennedy 2009). Netrin-1 exerts its effects through specific receptors such as deleted in colorectal cancer (DCC), neogenin (NEO), and uncoordinated-5 (UNC5) including UNC5a, UNC5b, UNC5c, and UNC5d (Layne et al. 2015). The identification of DCC and UNC5 receptors on cell types other than neurons supports the hypothesis that netrin-1 had additional functions besides the nervous system (Layne et al. 2015). Indeed, it has been reported that kidney proximal tubular epithelial-specific overexpression of netrin-1 inhibited inflammation and albuminuria in diabetic mice (Mohamed et al. 2012). Moreover, netrin-1 had a potential as a therapeutic target in several diabetic vascular complications (Carmeliet \& Tessier-Lavigne 2005, Layne et al. 2015). The present insight into the modulatory actions of netrin-1 on vascular function was mostly limited to vascular endothelial cells and smooth muscle cells (Fan et al. 2008, Brunet et al. 2014, Gabriella et al. 2015, Toque et al. 2017, Xing et al. 2017). It remains ill-defined whether netrin-1 is involved in sympathetic neurons paracrine signaling to regulate AFs function.

In the current study, we hypothesized that the secretion of netrin-1 from the perivascular sympathetic neurons decreased under hyperglycemia and eventually augmented the vascular adventitial remodeling. In this in vitro study, we utilized conditioned medium from the superior cervical ganglia (SCG) neurons to incubate with AFs. In vivo, vascular remodeling was produced in the T2DM rats that were fed with high-fat diet (HFD) combined with low-dose streptozotocin (STZ) administration. In terms of mechanism, it was investigated whether the UNC5b/cAMP/PKA/Nox4 pathway was implicated in the netrin-1 transduction signal in AFs.

\section{Materials and methods}

\section{Primary cell culture of AFs and SCG neurons}

The primary vascular AFs were cultured according to our previous study (Li et al. 2019). The AFs were isolated from the thoracic aorta of 8-week-old male Sprague-Dawley (SD) rats. Rats were obtained from Qinglongshan Lab Animal Ltd, Nanjing, China. Animal handling and experimental procedures were approved by the Ethics Committee of China Pharmaceutical University, in accordance with the Guidelines of Animal Experiment set by the Bureau of Sciences and Techniques of Jiangsu Province, China (NO.SYXK2007-0025).

Briefly, aortas were removed from rats after anesthesia. The peripheral fat was gently cleaned and then the media and intima layer were scraped off and the adventitia was cut into small pieces in DMEM (Gibco). The adventitia pieces were digested with $0.2 \%$ collagen I (Beyotime Biotechnology, China) for $5 \mathrm{~h}$ and then were centrifuged at $137 \boldsymbol{g}$ for 5 min. DMEM containing 10\% fetal bovine serum (Gibco), $100 \mathrm{U} / \mathrm{mL}$ penicillin and streptomycin (Hyclone, Beijing, China) was used to culture the sediment at $37^{\circ} \mathrm{C}$ with $5 \% \mathrm{CO}_{2}$. After $8-10$ days, the AFs grew out and reached $80-90 \%$. AFs in passage three to seven were used in our current experiments. AFs were identified according to their morphology features and immunocytochemistrical staining of marker proteins. Vimentin (1:200, Abways, CY5134) and $\alpha$-smooth muscle actin ( $\alpha$-SMA, 1:200, Wanleibio, WL02510-020) were used as positive and negative markers of AFs, respectively (Stenmark et al. 2013). 
The primary postganglionic sympathetic neurons were collected from the SCG of 1-to-3-day-old SD rat pups. SCG neurons provided a well-studied model of autonomic nervous system and exhibited the same characteristics as human unmyelinated sympathetic neurons. Under a dissecting microscope, the connective tissue and the paratracheal muscle were separated and then the ' $\mathrm{Y}$ ' branch of the common carotid artery was observed. Microscopic tweezer was used to carefully grasp the SCG to quickly remove it from the ' $\mathrm{Y}$ ' branch. The SCG were gently cleaned and enzymatically dissociated for $30 \mathrm{~min}$ at $37^{\circ} \mathrm{C}$ in $0.25 \%$ trypsin and then were centrifuged at $137 \boldsymbol{g}$ for $5 \mathrm{~min}$. Dissociated cells were applied to polylysine (Sigma)-and-laminin (Sigma)coated dishes and grown in DMEM (Gibco) supplemented with $50 \mathrm{ng} / \mathrm{mL}$ NGF (Sigma), 2\% B27 (Invitrogen), $2 \mathrm{mM}$ glutamine (Invitrogen), $100 \mathrm{U} / \mathrm{mL}$ penicillin and streptomycin (Hyclone) at $37^{\circ} \mathrm{C}$ with $5 \% \mathrm{CO}_{2}$. For mass cultures, neurons were plated at a density of eight ganglia per well in 6-cm dishes (for collection of conditioned medium) or one ganglion per $13-\mathrm{mm}$ glass slide (for immunofluorescence analysis). One day after plating, neurons were treated with antimitotic agent cytosine$\beta$-D-arabinoside ( $1 \mu \mathrm{M}$, Sigma) to prevent the growth of nonneuronal cells. After 3 days, neurons were washed with PBS twice to remove the antimitotic agent and used for our experiments after 3-4 days. SCG neurons were identified according to their morphology features and immunocytochemistrical staining of marker protein. Tyrosine hydroxylase (TH) was the rate-limiting enzyme in catecholamine synthesis and thus was used as a marker for sympathetic neurons (Damon 2000).

\section{BrdU incorporation assay}

Bromodeoxyuridine (BrdU) incorporation assay was performed as described previously (Wei et al. 2019). Briefly, AFs cultured in six-well plates were incubated with BrdU (10 $\mu \mathrm{M}$, NSolarbio, China) for $24 \mathrm{~h}$ in $5 \%$ $\mathrm{CO}_{2}-95 \% \mathrm{O}_{2}$ at $37^{\circ} \mathrm{C}$. After fixed by $4 \%$ paraformaldehyde solution, perforated by $0.3 \%$ Triton $\mathrm{X}$ and denatured by $2 \mathrm{M} \mathrm{HCl}$, AFs were incubated with anti-BrdU primary antibody (1:100, Proteintech ${ }^{\mathrm{TM}}$, China, 66241-1-Ig) overnight, followed by Cy3 (1:100, Proteintech ${ }^{\mathrm{TM}}$, China, SA00009-1). The immunofluorescence images were taken by fluorescence microscope (Carl Zeiss, scope A1). The percentage of BrdU-positive cells to the total amount of cells was calculated by ImageJ software (NIH).

\section{Cell migration assay}

Cell migration assay was detected by wound-healing assay and transwell migration assay as we described previously (Li et al. 2019). In the wound-healing assay, AFs were added into six-well plates for $12 \mathrm{~h}$. Then sterile toothpicks were used to make wounds. After washing with PBS three times, AFs were cultured with conditioned medium (CM). The wound area was photographed with a light microscope (magnification, 40x) and photos were taken again after $24 \mathrm{~h}$. In the transwell migration assay, AFs suspension $(200 \mu \mathrm{L})$ was added into the upper compartment of the chamber of transwell system (8-mm pore size, Corning Costar, USA) and CM with 10\% fetal bovine serum $(600 \mu \mathrm{L})$ containing different drugs was added into the bottom chamber. After incubating for $6 \mathrm{~h}$, cotton swabs were used to gently wipe off the cells in the upper layer of the membrane (not migrated). The migrated cells were fixed on the lower membrane with methanol for $20 \mathrm{~min}$ and then stained with $0.2 \%$ crystal violet for $30 \mathrm{~min}$. The number of migrated cells was calculated under a light microscope (magnification, 100x).

\section{Western blotting}

The protein expressions were detected via Western blot as we described previously (Li et al. 2018). The AFs were washed twice by PBS and then scraped in RIPA lysis buffer (BU Technology CO, LTD) with protease inhibitor cocktail (MedChemExpress, China) at 1:100 (v/v) dilution. Protein concentrations were analyzed via the $\mathrm{BCA}$ protein quantitative kit (BU Technology CO, LTD). $20 \mu$ g proteins were separated by $10 \%$ SDS-PAGE at $80 \mathrm{~V}$ for $30 \mathrm{~min}$ and then $120 \mathrm{~V}$ for $60 \mathrm{~min}$. Then proteins were transferred to polyvinylidene fluoride membranes (PVDF membrane, $0.45 \mu \mathrm{m}$, Millipore Co. Ltd.) and blocked with non-fat milk (5\% w/v) in Tris-buffered saline with Tween 20 for $90 \mathrm{~min}$. The membranes were probed with the primary antibodies of PCNA (1:1000; Abways, China, AB0051), collagen I (1:1000; Wanleibio, China, WL01931-020), Nox1 (1:1000; Abways, China, AY4775), Nox2 (1:1000; Abways, China, CY5739), Nox4 (1:1000; Abways, China, CY5255), p22phox (1:500; Santa Cruz, USA, sc-130550), p-PKA (thr-197) (1:500; CST, USA, 5661T), and PKA (1:500; Santa Cruz, USA, sc-390548) overnight at $4^{\circ} \mathrm{C}$ and then incubated with the secondary antibody at room temperature for $90 \mathrm{~min}$. GAPDH (1:5000, Abways, China, $\mathrm{AB} 0037)$ and $\beta$-actin (1:3000, Abways, China, AB0035) were used as control proteins. The blot was detected by chemiluminescent detection systems with LumiGlo and 
Peroxide (1:1, Tanon, China). Densitometric analysis of the images was performed with ImageJ software (NIH).

\section{Collection of conditioned medium (CM)}

SCG neurons were incubated in DMEM (5.5 mM glucose) which was supplemented with sterile D-glucose to achieve final glucose concentration of $25 \mathrm{mM}$ for 1 week, which was always used to mimic hyperglycemia condition in vitro study as previously described (Campanucci et al. 2010). MTT assay demonstrated that $25 \mathrm{mM} \mathrm{HG}$ had no significant influence on neuron viability. After washing SCG neurons with PBS twice, new DMEM $(5.5 \mathrm{mM}$ glucose) replaced original media for $24 \mathrm{~h}$ to eliminate the effect of NGF, B27, and glutamine on AFs. The supernatant was harvested, centrifuged at $137 \boldsymbol{g}$ for $5 \mathrm{~min}$ to remove cell debris, and stored at $-80^{\circ} \mathrm{C}$ until used.

\section{ELISA analysis for netrin-1 and cAMP}

The levels of netrin-1 and cAMP were analyzed with rat netrin-1 ELISA Kit (Angle Gene, China, ANG-E11172R) and rat cAMP ELISA Kit (Angle Gene, China, ANG-E11140R), respectively. The samples were collected and centrifuged after the incubation, and then the levels of netrin-1 and cAMP were measured according to manufacturer's instructions. The absorbance value was read at $450 \mathrm{~nm}$ by a microplate reader (POLARstar Omega ${ }^{\circledR}$, BMG,Germany). The concentrations were calculated according to the standard curve.

\section{RT-PCR for netrin-1 receptor}

Total RNA was isolated from the cultured cells by RNA extraction kit (TianGen, China). The concentration of the total RNA was analyzed by Nano100 ultramicro spectrophotometer (Allsheng, China). $1 \mu \mathrm{g}$ RNA was reverse-transcribed into cDNA in $20 \mu \mathrm{L}$ reaction volumes via the Fast Quant RT kit (TianGen, China). SYBR Green reaction mix (TianGen, China) in the $20 \mu \mathrm{L}$ reaction mixtures was used to perform real-time PCR by Real-Time qPCR instrument (Life Tech, QuantStudio 3).
The reaction was run at $95^{\circ} \mathrm{C}$ for $15 \mathrm{~min}$, followed by 40 cycles of $10 \mathrm{~s}$ at $95^{\circ} \mathrm{C}$ and $30 \mathrm{~s}$ at $60^{\circ} \mathrm{C}$. The mRNA levels were calculated by $\Delta \Delta \mathrm{CT}$ method and normalized by GAPDH as previously described (Vandesompele et al. 2002, Delloye-Bourgeois et al. 2009). The PCR primers (Sangon Biotech, China) used were listed in Table 1.

\section{RNA interference of netrin-1, UNC5b and Nox4}

siRNA of netrin-1, UNC5b, and Nox4 were purchased from Santa Cruz (sc-270422), Sangon Biotech, and Santa Cruz (sc-41586), respectively. The targeting sequence was as follows:Netrin-1 (5'-AAGCUGGACGCAGCAUGAUGC-3'), Nox4 (5'-AAUUCUCCGAA CGUGUCACGU-3'), and UNC5b (sense: 5'-CCUCAAGAUCAAGGUCUAUTT-3', antisense: 5'-AUAGACCUUGAUCUUGAGGTT-3'). The scrambled small RNA (5'-AAUUCUCCGAA CGUGUCACGU-3') was used as control in this study. The transfection of siRNA and scramble RNA was performed using Lipofectamine 2000 (Invitrogen) according to the manufacturer's protocol which was confirmed to be effective by RT-PCR and Western blot analysis.

\section{Measurement of reactive oxygen species (ROS) production}

The measurement of ROS production was performed using the fluorescent probe dihydroethidium (DHE; Sigma, CAS 104821-25-2) and dichloro-dihydrofluorescein-diacetate (DCFH-DA; Sigma, CAS 4091-99-0) (Kung et al. 2015, Chen et al. 2016). AFs were cultured in six-well plates in $5 \% \mathrm{CO}_{2}-95 \% \mathrm{O}_{2}$ at $37^{\circ} \mathrm{C}$. After treatment, each well was washed with $2 \mathrm{~mL}$ PBS two times, meanwhile $10 \mu \mathrm{M}$ DHE ( $2 \mathrm{~mL}$ ) or $10 \mu \mathrm{M}$ DCFH-DA $(2 \mathrm{~mL}$ ) per well was used to incubate AFs for $30 \mathrm{~min}$ in $5 \% \mathrm{CO}_{2}$ at $37^{\circ} \mathrm{C}$ without light. After the incubation, AFs were washed with $2 \mathrm{~mL}$ PBS three times, and fluorescence images were observed by fluorescent microscopy (Olympus IX53, Olympus). The fluorescent intensity was analyzed by ImageJ software, and the ratio of fluorescent intensity to that at basal level was quantified to represent ROS level.

Table 1 RT-PCR primer sequences.

\begin{tabular}{ll}
\hline UNC5a & 5'-TGTGCCTCTTCTTGCTGTTGCTG-3' \\
UNC5b & 5'-ATACCACTGTCCATCCGCCA-3' \\
UNC5c & 5'-TGGCAGGTGGACTTCGTGGAG-3' \\
UNC5d & 5'-GCTGCATGTCCTGTAACTCCTTGG-3' \\
DCC & 5'-CTCGGAATCCAGCCAGCACAAG-3' \\
NEO & 5'-TACCAGCCAACCAGAACCTTCCTC-3' \\
GADPH & 5'-ACAGTCAGCCGCATCTTCTT-3' \\
\hline
\end{tabular}

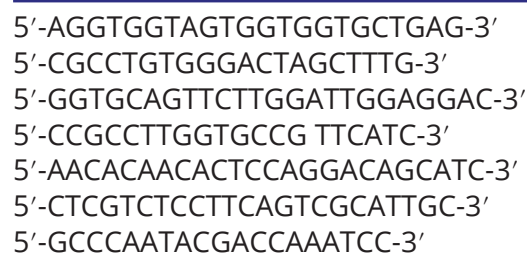

DCC receptor, deleted in colorectal cancer receptor; NEO receptor, neogenin receptor; UNC5 receptor, uncoordinated-5 receptor.
(C) 2020 Society for Endocrinology Published by Bioscientifica Ltd. Printed in Great Britain 


\section{Measurement of $\mathrm{H}_{2} \mathrm{O}_{2}$ production}

Extracellular $\mathrm{H}_{2} \mathrm{O}_{2}$ was measured by Amplex Red (Invitrogen) as previously described (Takac et al. 2011). AFs were washed with PBS twice and subsequently incubated in a working solution containing $100 \mu \mathrm{M}$ Amplex Red and 0.25 units/mL HRP without light. After 30 min, the supernatant was collected and the $\mathrm{H}_{2} \mathrm{O}_{2}$ dependent oxidation of Amplex Red was measured in a microplate fluorometer (FL-600, BioTek Instruments) at excitation $540 \mathrm{~nm}$ and emission 580. The level of $\mathrm{H}_{2} \mathrm{O}_{2}$ was quantified by a standard concentration curve.

Intracellular $\mathrm{H}_{2} \mathrm{O}_{2}$ was tested using $\mathrm{H}_{2} \mathrm{O}_{2}$ assay kit (Beyotime Institute of Biotechnology, China). Ferrous ions $\left(\mathrm{Fe}^{2+}\right)$ were oxidized to ferric ions $\left(\mathrm{Fe}^{3+}\right)$ by $\mathrm{H}_{2} \mathrm{O}_{2}$, and then formed a purple complex with an indicator dye, xylenol orange (3,3'-bis [N,N-di(carboxymethyl)-aminomethyl]o-cresolsulfone-phthalein, disodium salt, and xylenol orange), which was read at $560 \mathrm{~nm}$ by a microplate reader (POLARstar Omega ${ }^{\circledR}$, BMG, Germany) (Deiana et al. 1999). The level of $\mathrm{H}_{2} \mathrm{O}_{2}$ was calculated according to a standard concentration curve originated from standard solutions.

\section{Confocal microscopy for the co-localization of Nox4 and p22phox in AFs}

Immunofluorescent assay was used to co-localize Nox4 and p22phox. AFs were fixed with $4 \%$ paraformaldehyde solution for $15 \mathrm{~min}$ at room temperature and then permeabilized with $0.3 \%$ Triton-X for $15 \mathrm{~min}$. After washing with PBS three times for 5 min each time, cells were incubated with 5\% BSA for $1 \mathrm{~h}$ and incubated with the primary antibodies of Nox4 (1:200; Abways, China, CY5255) or p22phox (1:200; Santa Cruz, sc-130550) overnight at $4^{\circ} \mathrm{C}$. After washing with PBS three times, the cells were stained with secondary antibodies conjugated to Alexa 594 (1:200; Abways China, AB0151) or Alexa 488 (1:200; Abways China, AB0141) for $2 \mathrm{~h}$ at room temperature. Then cells were washed with PBS and visualized using a confocal microscopy (Carl Zeiss), and the images were processed by ZEN blue 2.3 software (Carl Zeiss). The summarized co-localization efficiency data were expressed as Pearson correlation coefficient (PCC).

\section{Establishment of rat T2DM mode}

Adult male Sprague-Dawley (SD) rats, weighing 130-150 g, were obtained from Qinglongshan Lab Animal Ltd, Nanjing, China. Animal handling and experimental procedures were approved by the Ethics Committee of China Pharmaceutical University and conducted according to the NIH Guide for the Care and Use of Laboratory Animals.

SD rats were developed as a rat model of T2DM according to the method in our previous study (Li et al. 2018). Rats were fed with an HFD (4.7 kcal/g) consisting (percentage of total kcal) $44 \%$ fat, $37 \%$ carbohydrate, and $19 \%$ protein or a control ordinary chow $(3.8 \mathrm{kcal} / \mathrm{g})$ consisting (percentage of total kcal) 10\% fat, 69\% carbohydrate, and $21 \%$ protein. Both the HFD and control ordinary chow were purchased from Qinglongshan Lab Animal Ltd. SD Rats were divided into three groups $(n=6$ rats per group), control (regular chow), untreated diabetes (fed with HFD following an injection of low STZ), diabetes treated with recombinant netrin-1 $(120 \mu \mathrm{g} / \mathrm{kg} / \mathrm{per} 48 \mathrm{~h}$, intraperitoneally) (Ranganathan et al. 2015). Recombinant soluble netrin-1 was purchased from R\&D Systems, USA (1109-N1-025/CF).

In the first 6 weeks, rats in both untreated and treated diabetes group were given HFD alone and rats in the control group only received regular chow. On the beginning of seventh week, rats fed with HFD received a single time intraperitoneal injection of STZ $(40 \mathrm{mg} / \mathrm{kg}$, dissolved in $\mathrm{pH} 4.5$ citrate buffer). Control rats only received an equivalent volume of citrate buffer. After the injection of STZ, all groups maintained their original diets. Rats on HFD with low STZ were recognized as diabetic rats while the fasting blood glucose (FBG) level reached $16.7 \mathrm{mM}$ after 1 week of STZ injection. In weeks 8-13, the treatment with netrin-1 was conducted every other day. However, rats in the groups of control and untreated diabetes were given an equal volume of saline solution. A gain of body weight was monitored at an interval of 1 week.

The serum was collected and stored at $-20^{\circ} \mathrm{C}$ before use. FBG, fasting insulin, malondialdehyde (MDA), and superoxide dismutase (SOD) were assayed following the instructions of the kits provided by the Nanjing Jiancheng Bio engineering Institute (China). Homeostasis model assessment of insulin resistance (HOMA-IR) was calculated as FBG $(\mathrm{mmol} / \mathrm{L}) \times$ fasting insulin $(\mathrm{mU} / \mathrm{L}) / 22.5$.

\section{Immunofluorescent staining of aortic rings}

The immunofluorescent staining was used for co-localization of netrin-1 and sympathetic nerve fibers or adventitia. Briefly, the aortic rings were fixed in OCT embedding matrix for frozen sections. Cross-sections from the mid-portion of the aorta were achieved by a cryostat at a thickness of $10 \mu \mathrm{m}$. After fixed in cold acetone for 
10 min and permeabilized in $0.3 \%$ Triton-X for $15 \mathrm{~min}$, sections were blocked for $1 \mathrm{~h}$ with 5\% BSA. Sections were incubated with the primary antibodies of TH (1:200; Santa Cruz, sc-73152), ER-TR7 (1:200; Santa Cruz, sc-73355), or netrin-1 (1:200; Affinity, USA, DF8579), or UNC5b (1:200; Abcam, ab189914) overnight at $4^{\circ} \mathrm{C}$. After washing with PBS for 3 times for $5 \mathrm{~min}$, the sections were incubated with the secondary antibodies of Alexa 594 (1:200; Abways, China, AB0151) or Alexa 488 (1:200; Abways, China, AB0141) for $1 \mathrm{~h}$ at room temperature. Negative controls omitted the primary antibody. Fluorescent images were photographed by a fluorescence microscope (Carl Zeiss, Scope A1) The summarized colocalization efficiency data were expressed as Pearson correlation coefficient (PCC).

\section{Immunohistochemistry for PCNA and Nox4}

Immunohistochemistry was performed as we described previously (Li et al. 2018). The aortic rings were fixed in $4 \%$ paraformaldehyde and embedded in paraffin. The paraffin sections of the aortic rings were sliced, dewaxed, and washed with PBS three times for 5 min, followed by blocked with $3 \% \mathrm{H}_{2} \mathrm{O}_{2}$ for $10 \mathrm{~min}$ and 5\% BSA for $60 \mathrm{~min}$ at room temperature. For immunohistochemical studies, the sections were incubated with anti-PCNA (1:200, Abways, China, AB0051) and anti-Nox4 (1:200, Abways, China, CY5255) at $4^{\circ} \mathrm{C}$ overnight. After washing with PBS three times, each section was incubated with the secondary antibody (1:500, Abways, China, AB0101) for 90 min at room temperature. After washing with PBS three times, sections were added to 50-100 $\mu \mathrm{L}$ Diaminobenzidine solution (Beyotime Biotechnology, China) for $10 \mathrm{~min}$. Next, sections were washed with distilled water and stained with hematoxylin for $25 \mathrm{~s}$. The sections were examined under Olympus CX31 microscope. Mean integrated optical density (IOD) was determined by Image Pro Plus 6.0 software (Media Cybernetics, Inc. USA). The adventitial proliferation index was evaluated by the extent of adventitial PCNA expression.

\section{HE staining and morphological analysis}

After deparaffinization and rehydration, sections were dyed with hematoxylin solution (hematoxylin $1 \mathrm{~g}$, sodium iodate $0.2 \mathrm{~g}$, aluminum potassium sulfate, $50 \mathrm{~g}$, citric acid $1 \mathrm{~g}$, chloral hydrate $50 \mathrm{~g}$, distilled water added to $1 \mathrm{~L}$ ) for $10 \mathrm{~min}$, and then alcoholic eosin $(2.5 \mathrm{~g}$ eosin, $500 \mathrm{~mL}$ distilled water, hydrochloric acid $10 \mathrm{~mL}$, filter product dissolved in $1000 \mathrm{~mL}$ 95\% alcohol, double diluted before use) for $30 \mathrm{~s}$. The vascular adventitia remodeling indexes were measured by Image Pro Plus 6.0 software (Media Cybernetics, Inc. USA). The adventitial area indexes were represented as adventitial area/vessel wall area, and the adventitial thickness index was represented as adventitia thickness/vessel wall thickness. The adventitial fibrosis index was evaluated by adventitial collagen deposition, which was measured following the instructions of the Masson kit provided by Sbjbio (Nanjing, China).

\section{Statistical analysis}

Statistical analysis was performed with IBM SPSS Statistics 20 software. Data are presented as means \pm s.E.M. of independent experiments. Significant differences between and within multiple groups were examined using ANOVA for repeated measures, followed by Duncan's multiple range test. The Independent-Samples $t$-test was used to detect significant differences between two groups. $P<0.05$ was considered statistically significant.

\section{Results}

\section{Netrin-1 deficiency in SCG neurons contributed to AFs remodeling}

As shown in Supplementary Fig. 1A (see section on supplementary materials given at the end of this article), under the light microscopy (the photographs were at 400× magnification), AF appeared spindle shaped or elongated. Immunocytochemistry showed that AFs positively expressed vimentin and negatively expressed $\alpha$-SMA, which identified the extracted AFs. SCG neurons were isolated from the superior cervical ganglia of SD rat pups. Under the light microscope, SCG neurons had clear, centrally located nuclei and continued to extend long and grow in size (Supplementary Fig. 1B). Immunocytochemistry showed $\mathrm{TH}$, a positive marker of sympathetic neurons, was positively expressed in the SCG neurons which identified the extracted sympathetic neurons.

ELISA assay showed that about $300 \mathrm{pg} / \mathrm{mL}$ netrin-1 was endogenously secreted by SCG neurons in the supernatant under physiological condition. To explore whether netrin-1 from SCG neurons played a role on AFs, SCG neurons were transfected with netrin-1 siRNA, which inhibited the protein expression of netrin-1 by $61.3 \%$ by Western blot assay (not shown). ELISA assay showed that the netrin-1 level in CM (netrin-1 siRNA) decreased to $100 \mathrm{pg} / \mathrm{mL}$ compared with CM (Control) (Fig. 1A). 
Vascular adventitial remodeling is characterized by AFs proliferation, migration, phenotypic transformation, and extracellular matrix collagen deposition. As shown in Fig. $1 \mathrm{~B}$, AFs were exposed to the supernatant collected from SCG neurons, and it was found that netrin-1 deficiency in the supernatant induced the protein expression of PCNA, a typical marker of proliferation by Western blot. Consistently, BrdU incorporation assay showed that netrin-1 deficiency in the supernatant induced AFs proliferation (Supplementary Fig. 2A and B). However, when the netrin-1 level in the supernatant was restored to $300 \mathrm{pg} / \mathrm{mL}$ by adding exogenous soluble recombinant netrin-1, AFs proliferation was completely blocked. Collagen-I and collagen-III are the major vascular collagen, while collagen-I is considered to be most relevant to arterial injury. As shown in Fig. 1C, exogenous netrin-1 (reaching a final concentration of $300 \mathrm{pg} / \mathrm{mL}$ ) also dramatically decreased the protein level of collagen-I.

In vascular injury, many AFs tend to transdifferentiate to myofibroblasts to migrate to the media and even the neointima. The wound-healing assay and the transwell assay were used to assess cell migration. Either the wound areas (Fig. 1D and E) or cell number underneath the membrane (Fig. 1F) dramatically increased in $\mathrm{CM}$ (netrin-1 siRNA)-incubated AFs, suggesting that netrin-1 (300 pg/mL) significantly inhibited AFs migration.

Therefore, the data indicated that netrin-1 from SCG neurons was one of key communication signal responsible for AFs function under physiological condition.

\section{Exogenous netrin-1 abolished CM(HG)-evoked AFs remodeling}

To confirm the effects of netrin-1 on vascular adventitia, AFs were exposed to $\mathrm{CM}(\mathrm{HG})$ which was collected from the supernatant of SCG neurons incubated with gradient HG (15-30 mM). As shown in Fig. 2A, B and Supplementary Fig. 2C, it was found that $25 \mathrm{mM}$ HG-treated neurons supernatant showed most significant stimulation on AFs proliferation and collagen synthesis. Meanwhile, the concentration of netrin-1 was tested in $\mathrm{CM}(\mathrm{HG})$ by ELISA assay. As shown in Fig. 2C, netrin-1 level in neuron supernatant dose-dependently decreased by gradient HG (15-30 mM), which was lowest under 25 mM HG. Therefore, netrin- 1 might be a key modulator from SCG neurons to regulate AFs function under hyperglycemia.

As shown in Fig. 2D, F, and Supplementary Fig. 2D, AFs continued collagen synthesis and proliferation after
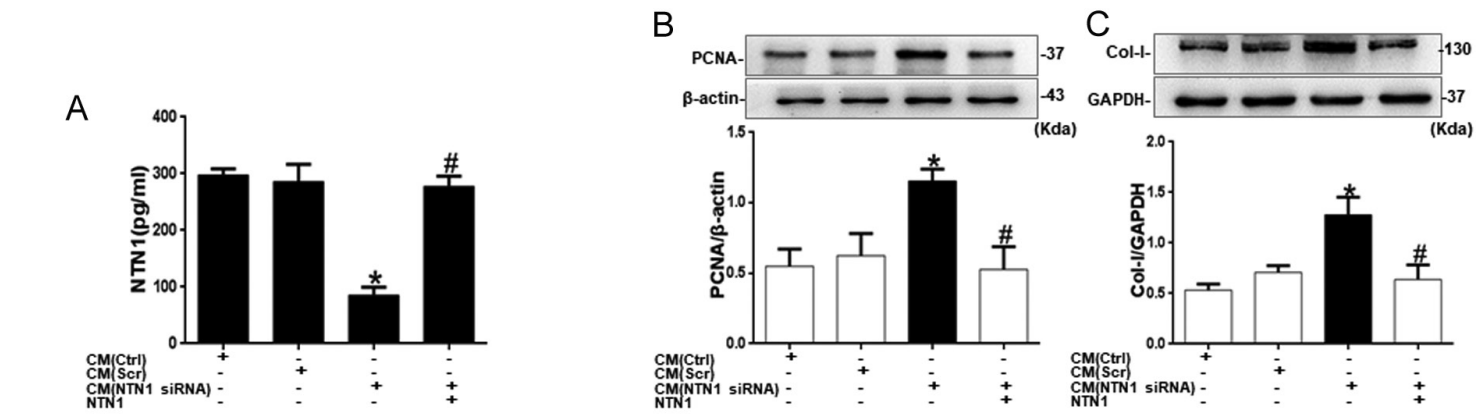

D

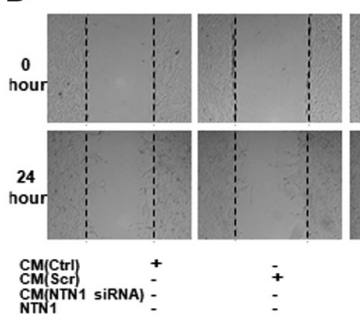

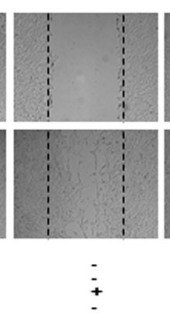
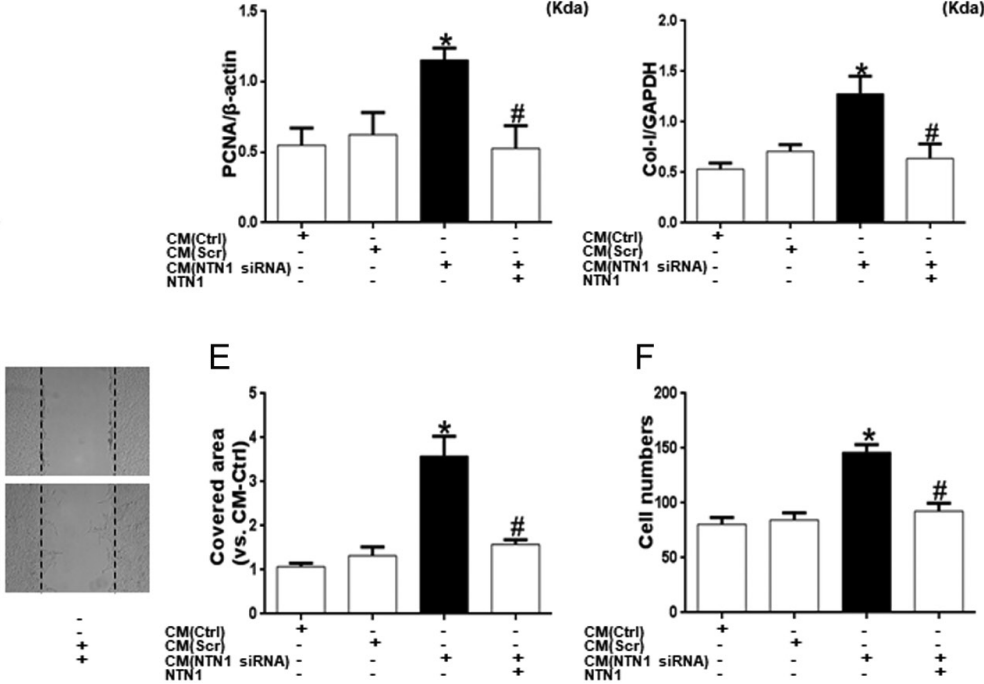

Figure 1

Netrin-1 deficiency in SCG neurons contributed to AFs remodeling. SCG neurons were transfected with netrin-1(NTN1) siRNA, and new DMEM (5.5 mM glucose) replaced the original media for $24 \mathrm{~h}$. The supernatant was harvested, centrifuged, and then named as conditioned medium (CM), and soluble recombinant netrin-1 was directly added into the supernatant to achieve final concentration of 300 pg/mL. SCG neurons were transfected with Scramble RNA (Scr) as a negative control. Summarized data showed the concentration of netrin-1 in the cell supernatant by ELISA assay (A). Summarized data showed AFs proliferation by the expression of PCNA (B) and collagen I (C), AFs migration with the wound healing assay, and the transwell migration assay ( $D, E$, and F). Data are means \pm S.E.M., $n=3$ replicates. ${ }^{*} P<0.05$ vs CM (Scr), ${ }^{2} P<0.05$ vs CM (NTN1 siRNA). 
and

A

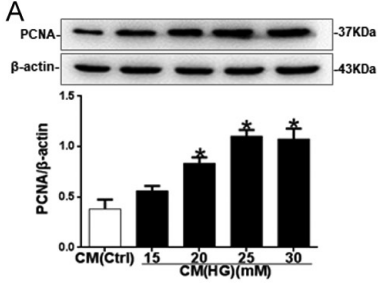

D

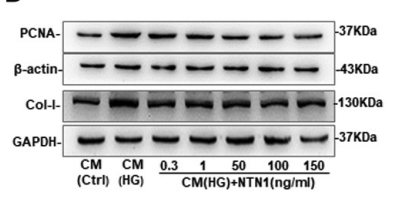

B
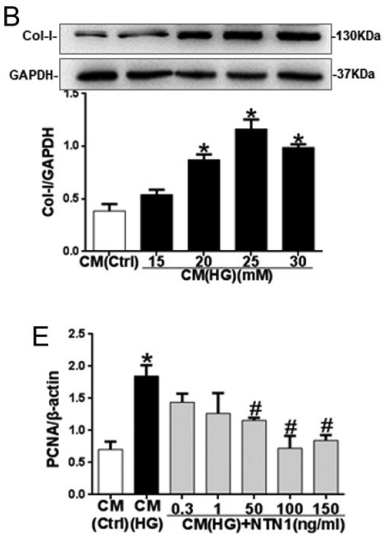

C
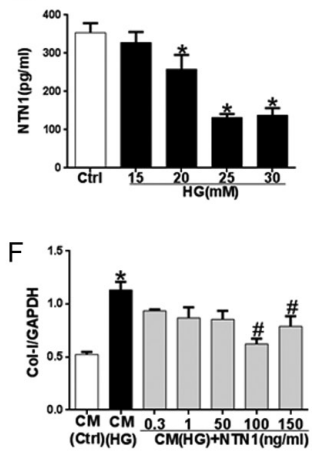

Figure 2

Exogenous netrin-1 abolished CM(HG)-evoked AFs proliferation and collagen synthesis. SCG neurons were incubated in DMEM supplemented with sterile D-glucose to achieve final glucose concentration of 15, 20, 25, and $30 \mathrm{mM}$ for 1 week. Then, new DMEM (5.5 mM glucose) replaced the original media for $24 \mathrm{~h}$. The supernatant was harvested and centrifuged to remove cell debris. AFs were incubated with $\mathrm{CM}(\mathrm{Ctrl})$ or $\mathrm{CM}(\mathrm{HG})$ for $24 \mathrm{~h}$. Representative Western blot gel documents and summarized data showed the expression of PCNA (A) and collagen I (B) in AFs. The concentration of netrin-1 was tested by ELISA assay (C). AFs were incubated with $\mathrm{CM}(\mathrm{HG})$ with different dose of netrin-1 $(0,0.3$, $1,50,100$, and $150 \mathrm{ng} / \mathrm{mL}$ ) for $24 \mathrm{~h}$.

Representative Western blot gel documents and summarized data showed the expression of PCNA and collagen I (D, E, and F) in AFs. Data are means \pm S.E.M., $n=3$ replicates. ${ }^{\star} P<0.05$ vs $\mathrm{CM}(\mathrm{Ctrl})$ or $\mathrm{Ctrl}, \# P<0.05$ vs $\mathrm{CM}(\mathrm{HG})$. the restoration of netrin-1 concentration to $300 \mathrm{pg} / \mathrm{mL}$, a normal physiological level. However, when the level of netrin-1 reached $100 \mathrm{ng} / \mathrm{mL}, \mathrm{CM}(\mathrm{HG})$-induced AFs proliferation and collagen synthesis were dramatically inhibited. In Supplementary Fig. 3A, B, C, D, and E, the BrdU-positive cell percent, the protein expression of collagen-I, the wound areas by wound-healing assay, and the average number of AFs in the lower side of the membrane by transwell assay markedly increased in $\mathrm{CM}(\mathrm{HG})$-incubated AFs, which were almost completely abolished by exogenous netrin-1 (100 ng/mL) with no significant effects on AFs viability (not shown).

These results suggested that netrin-1 was one of the key communication signal between SCG neurons and AFs under hyperglycemia condition. Moreover, netrin-1 at a pharmacological dose could inhibit AFs remodeling induced by hyperglycemia-stimulated SCG supernatant.

\section{UNC5b functioned as netrin-1 receptor in AFs}

To determine which receptor mediated the effect of exogenous netrin-1 on AFs, we tested the mRNA levels of netrin-1-specific receptors on AFs, including UNC5a, UNC5b, UNC5c, DCC, and NEO. As shown in Fig. 3A, the mRNA expression of UNC5b was much higher than others, indicating that UNC5b was the main netrin-1specific receptor expressed in AFs. For further verifying if UNC5b mediated the effects of exogenous netrin-1 on AFs, UNC5b siRNA was transfected in AFs which inhibited the expression of UNC5b mRNA by $67.19 \%$ (Fig. 3B). The co-immunoprecipitation indicated that netrin-1 indeed bound with UNC5b under CM(Ctrl) condition (not shown). As shown in Fig. 3C, D, E, F, and Supplementary Fig. 2E,
UNC5b gene deficiency reversed exogenous netrin-1mediated inhibition of $\mathrm{CM}(\mathrm{HG})$-induced proliferation, collagen synthesis, and migration in AFs. Collectively, it was suggested that exogenous netrin-1 inhibited AFs remodeling by binding UNC5b receptor on AFs.

The cAMP/PKA is a key pathway of netrin-1-UNC5b downstream signal transduction (Ming et al. 1997, Höpker et al. 1999, Komatsuzaki et al. 2002, Stork \& Schmitt 2002). To further explore how exogenous netrin-1 inhibited vascular remodeling on AFs after binding to UNC5b, we detected the intracellular cAMP production and PKA phosphorylation. Among the phosphorylation sites of PKA, the phosphorylation at the site of thr-197 is essential for the optimal biological activity of PKA (Cheng et al. 1998). As shown in Fig. 3G and H, exogenous netrin-1 significantly increased cAMP production and PKA phosphorylation at the site of thr-197 in AFs compared with $\mathrm{CM}(\mathrm{HG})$ group, while this therapeutic effect of exogenous netrin-1 was eliminated after silencing UNC5b, implicating that the cAMP/PKA pathway was netrin-1/UNC5b downstream signal pathway, which mediated the amelioration of vascular remodeling.

\section{Nox4 was regulated by netrin-1/UNC5b/cAMP/PKA pathway in AFs}

It was well established that vascular adventitial remodeling, especially caused by diabetes, was strongly associated with the presence of oxidative stress (Haurani \& Pagano 2007). Here, DHE and DCFH-DA staining were used to detect ROS level. As shown in Fig. 4A and Supplementary Fig. 4A, ROS production in AFs dramatically increased in response to $\mathrm{CM}(\mathrm{HG})$, which was inhibited by $\mathrm{N}$-acetylcysteine 


\begin{tabular}{l|l|l|l|l|}
\hline $\begin{array}{l}\text { Journal of } \\
\text { Endocrinology }\end{array}$ & H-F Wang et al. & $\begin{array}{l}\text { Netrin-1 inhibited adventitial } \\
\text { remodeling }\end{array}$ & $\mathbf{2 4 4 : 3}$ & $\mathbf{4 5 3}$ \\
\hline
\end{tabular}
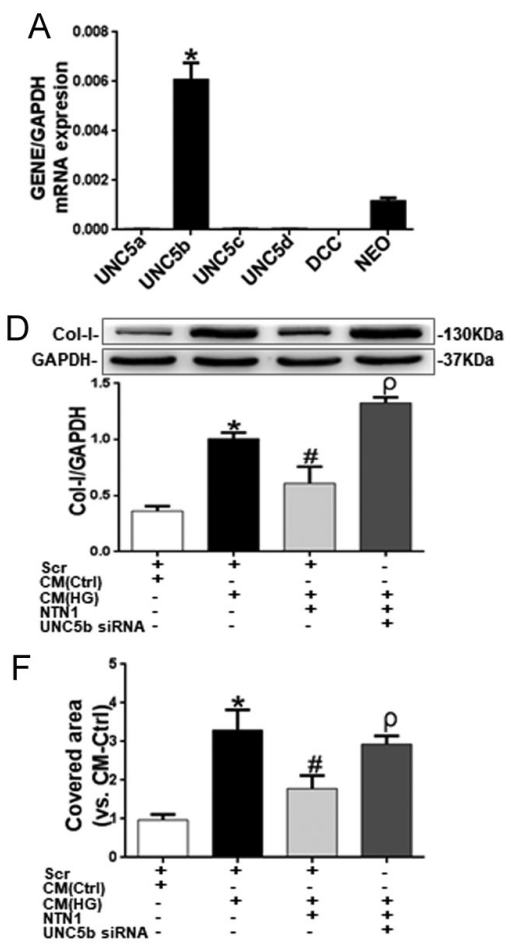

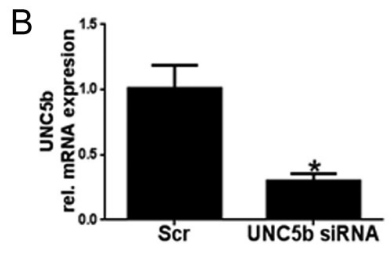

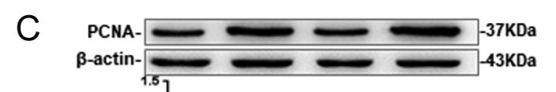

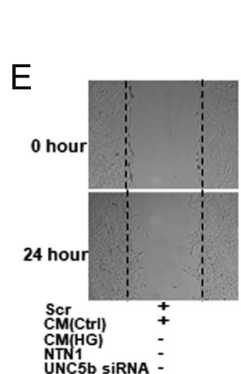

G

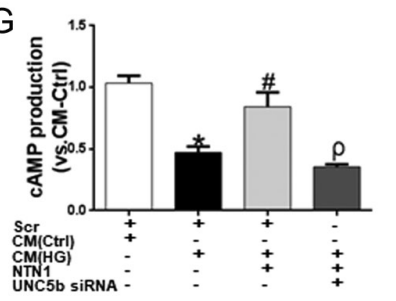

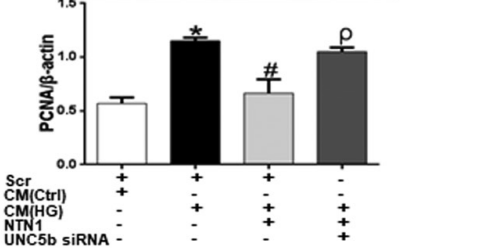
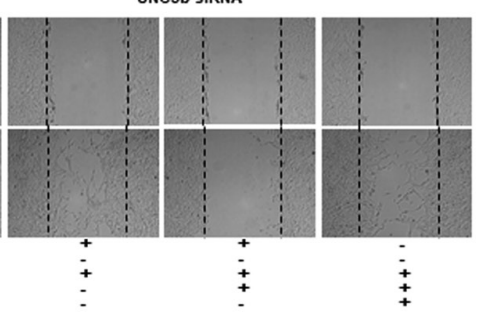

$\mathrm{H}$

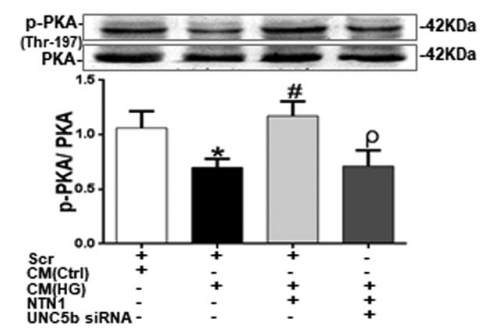

Figure 3

UNC5b functioned as netrin-1 receptor in Afs. mRNA expression of netrin-1 specific receptors on AFs by real-time RT-PCR (A). AFs were transfected with UNC5b siRNA, and mRNA expression of UNC5b in AFs was measured by real-time RT-PCR (B). UNC5b siRNA was transfected into AFs which were incubated with $\mathrm{CM}(\mathrm{HG})$ with or without netrin-1 $(100 \mathrm{ng} / \mathrm{mL})$ for $24 \mathrm{~h}$. Representative Western blot gel documents and summarized data showed the expression of PCNA(C) and collagen I (D) in AFs. The wound-healing assay was used to test cell migration and summarized data showed the scraped areas (E and F). The intracellular cAMP level was tested by ELISA assay (G) and the ratio of p-PKA/PKA was tested by Western blot (H). Data are means \pm S.E.M., $n=3$ replicates. ${ }^{*} P<0.05$ vs Neo group or Scramble RNA (Scr) or CM (Ctrl), $\# P<0.05$ vs CM (HG), $\rho P<0.05$ vs CM(HG) plus netrin-1 group.

(NAC), a ROS scavenger (Chen et al. 2016), suggesting that $\mathrm{CM}(\mathrm{HG})$ induced ROS production in AFs. NADPH oxidase has been identified as a major source of vascular ROS (Haurani \& Pagano 2007). CM(HG)-induced ROS production was reduced by apocynin, a NADPH oxidase inhibitor, indicating that the ROS production during $\mathrm{CM}(\mathrm{HG})$ stimulation was mainly originated from NADPH oxidase. Importantly, we found that exogenous netrin-1 significantly blocked the increase of intracellular ROS production induced by $\mathrm{CM}(\mathrm{HG})$.

To gain further insights into the molecular mechanisms underlying ROS reduction by exogenous netrin-1, the possible involvement of NADPH oxidase subtypes was examined. There are three NADPH oxidase isoforms present in rats AFs, namely Nox1, Nox2, and Nox4; however, Nox4 is the most predominant and abundant isoform in AFs (Haurani \& Pagano 2007, Barman et al. 2014). We examined the protein expression in Nox1, Nox2, and Nox4 in AFs treated with CM(HG) or netrin-1. Supplementary Figure $4 \mathrm{~B}$ showed that the protein level of Nox1 had no significant difference after the stimulation with different dose of $\mathrm{CM}(\mathrm{HG})$.
In Supplementary Fig. 4C, the protein expression of Nox2 was up-regulated by $\mathrm{CM}(\mathrm{HG})$, but showed no response to exogenous netrin-1. Supplementary Fig. 4D showed that Nox4 protein expression induced by $\mathrm{CM}(\mathrm{HG})$ was significantly attenuated by exogenous netrin-1. Taken together, Nox4 was critically implicated in exogenous netrin-1 transduction signal, and the abrogation of Nox4 at least partly explained exogenous netrin-1 inhibitory effect on ROS production.

To further verify if Nox 4 was a downstream molecular of the netrin-1/UNC5b/cAMP/PKA pathway in vascular adventitial remodeling, UNC5b siRNA and H-89, a PKA inhibitor, were used, respectively. It is reported that Nox4 is constitutively active, so the overall ROS output of Nox4 may be directly controlled by its expression level (Prior et al. 2016). UNC5b siRNA transfection (Fig. 4B, D and E) and H-89 treatment (Fig. 4C, F and G) abolished the suppression of exogenous netrin-1 on $\mathrm{CM}(\mathrm{HG})$-induced increase of Nox 4 and p22phox. It is essential for Nox4 activation that p22phox directly binds Nox4 (Prior et al. 2016); thus, the co-localization of Nox4 and p22phox was detected by confocal microscopy. Yellow punctuates or 


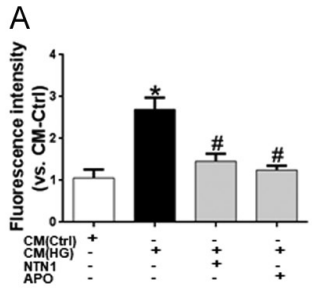

E

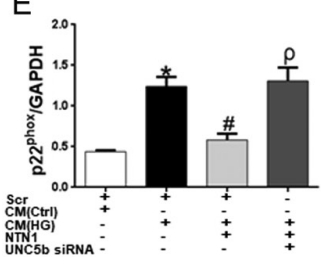

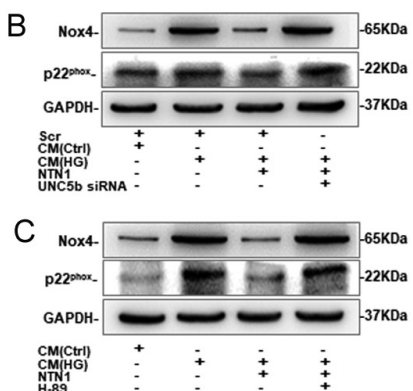

$\mathrm{F}$

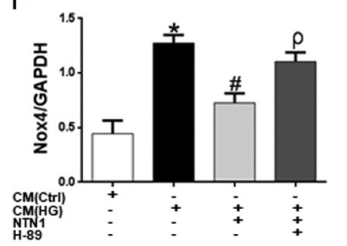

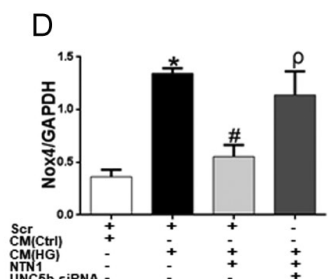

G

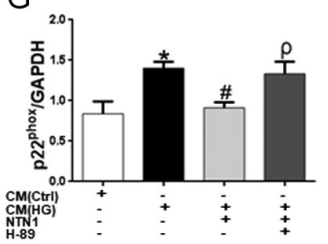

Figure 4

Nox4 was regulated by netrin-1/UNC5b/cAMP/ PKA pathway in AFs. AFs were incubated with $\mathrm{CM}(\mathrm{HG})$ and treated with netrin-1 $(100 \mathrm{ng} / \mathrm{mL})$ or apocynin (APO, $10 \mu \mathrm{M}$ ) for $24 \mathrm{~h}$. Summarized data (A) showed ROS production in AFs stained by DHE. AFs were incubated with $\mathrm{CM}(\mathrm{HG})$ and treated with netrin-1 $(100 \mathrm{ng} / \mathrm{mL})$ for $24 \mathrm{~h}$ in the presence or absence of UNC5b siRNA or $\mathrm{H}-89(10 \mu \mathrm{M})$. Representative Western blot gel documents and summarized data showed the expression of Nox4 and p22phox in AFs (B, C, D, E, F, and G). Data are means \pm S.E.M., $n=3$ replicates. $* P<0.05$ vs $\mathrm{CM}(\mathrm{Ctrl}), \# P<0.05$ vs $\mathrm{CM}(\mathrm{HG}), \mathrm{\rho} P<0.05$ vs $\mathrm{CM}(\mathrm{HG})$ plus netrin-1 group. patches which merged by red ones (Nox4) and green ones (p22phox) in cytoplasmic regions represented the aggregation of Nox4 and p22phox in AFs (Supplementary Fig. 4E and F). Compared with $\mathrm{CM}(\mathrm{HG})$ group, yellow dots were barely detected in AFs treated with exogenous netrin-1. It suggested that exogenous netrin-1 inhibited the assembled of Nox4 and p22phox. Nevertheless, after treated with UNC5b siRNA or H-89, the inhibition of exogenous netrin-1 on Nox4 activation was reversed, indicating that Nox 4 was a downstream signal of netrin-1/ UNC5b/cAMP/PKA pathway.

In contrast to Nox1 and Nox2, Nox4 primarily emits $\mathrm{H}_{2} \mathrm{O}_{2}$ instead of $\mathrm{O}_{2} \cdot-$ due to a highly conserved histidine residue in the E-loop of Nox4 (Takac et al. 2011). The production of both intra and extracellular $\mathrm{H}_{2} \mathrm{O}_{2}$ was elevated by $\mathrm{CM}(\mathrm{HG})$ and then was reduced after treatment of exogenous netrin- 1 comparable to catalase, an inhibitor of $\mathrm{H}_{2} \mathrm{O}_{2}$, indicating that netrin-1 inhibited oxidative stress by diminishing $\mathrm{H}_{2} \mathrm{O}_{2}$ (Supplementary Fig. $5 \mathrm{~A})$. Additionally, we found that RNAi of UNC5b blocked the therapeutic action of exogenous netrin-1 on $\mathrm{CM}(\mathrm{HG})$ induced production of both intra- and extracellular $\mathrm{H}_{2} \mathrm{O}_{2}$ (Supplementary Fig. 5C), indicating that $\mathrm{H}_{2} \mathrm{O}_{2}$ existed in the downstream of netrin-1/UNC5b signaling. Further, $\mathrm{CM}(\mathrm{HG})$-induced $\mathrm{H}_{2} \mathrm{O}_{2}$ production was reduced by Nox4 siRNA, which inhibited the protein expression of Nox 4 by $69.22 \%$ (Supplementary Fig. 5B), indicating that the $\mathrm{H}_{2} \mathrm{O}_{2}$ production during $\mathrm{CM}(\mathrm{HG})$ stimulation was mainly derived from Nox4 (Supplementary Fig. 5C). Besides, $\mathrm{H}_{2} \mathrm{O}_{2}$ dose-dependently exacerbated vascular remodeling as shown in the increase of the BrdU positive cell percent and protein expression of collagen-I as well as cell migration (Supplementary Fig. 5D, E, F, G, and $\mathrm{H})$. Therefore, $\mathrm{H}_{2} \mathrm{O}_{2}$ was a downstream signaling of
netrin-1/UNC5b/cAMP/PKA/Nox4 pathway to play a key role in vascular adventitial remodeling in AFs.

\section{Netrin-1 attenuated vascular adventitia remodeling in T2DM rats}

Following an injection of STZ in the seventh week, HOMA-IR and FBG dramatically increased compared with the control group (Fig. 5A and B). Therefore, a T2DM rat model was successfully established in vivo study. However, the intervention with netrin-1 in the last 6 weeks had no significant influence on HOMA-IR and FBG. The level of oxidative stress in vivo was measured by the level of serum MDA, a marker for oxidative stress and SOD activity, an anti-oxidase. As shown in Fig. 5C and $\mathrm{D}$, the elevated level of MDA and the decreased activity of SOD in T2DM rats were significantly alleviated by netrin-1 intervention.

Fibroblasts had an even closer neighborhood to the nerve varicosities than smooth muscle cells as demonstrated by the mean values of the distances between the nerve varicosities and each of the cell types (SoaresDa-Silva \& Azevedo 1985). In immunofluorescent assay, AFs and sympathetic neurons were labeled by antibody ER-TR7 and TH, respectively. As shown in Supplementary Fig. 6A, the sympathetic neurons (red color) were confined to the adventitial side of the media muscle coat and surrounded the periphery of the aorta. The co-localization of netrin-1 and ER-TR7 or TH further confirmed that netrin-1 existed in sympathetic neurons and vascular adventitia (Fig. 5E). As shown in Supplementary Fig. 6B, C, and $\mathrm{D}$, histomorphometry analysis revealed that diabetes induced dramatic adventitia thickening compared with the control group, indicating the vascular adventitia 


\begin{tabular}{l|l|l|r|r|}
$\begin{array}{l}\text { Journal of } \\
\text { Endocrinology }\end{array}$ & H-F Wang et al. & $\begin{array}{l}\text { Netrin-1 inhibited adventitial } \\
\text { remodeling }\end{array}$ & $\mathbf{2 4 4 : 3}$ & $\mathbf{4 5 5}$ \\
\hline
\end{tabular}
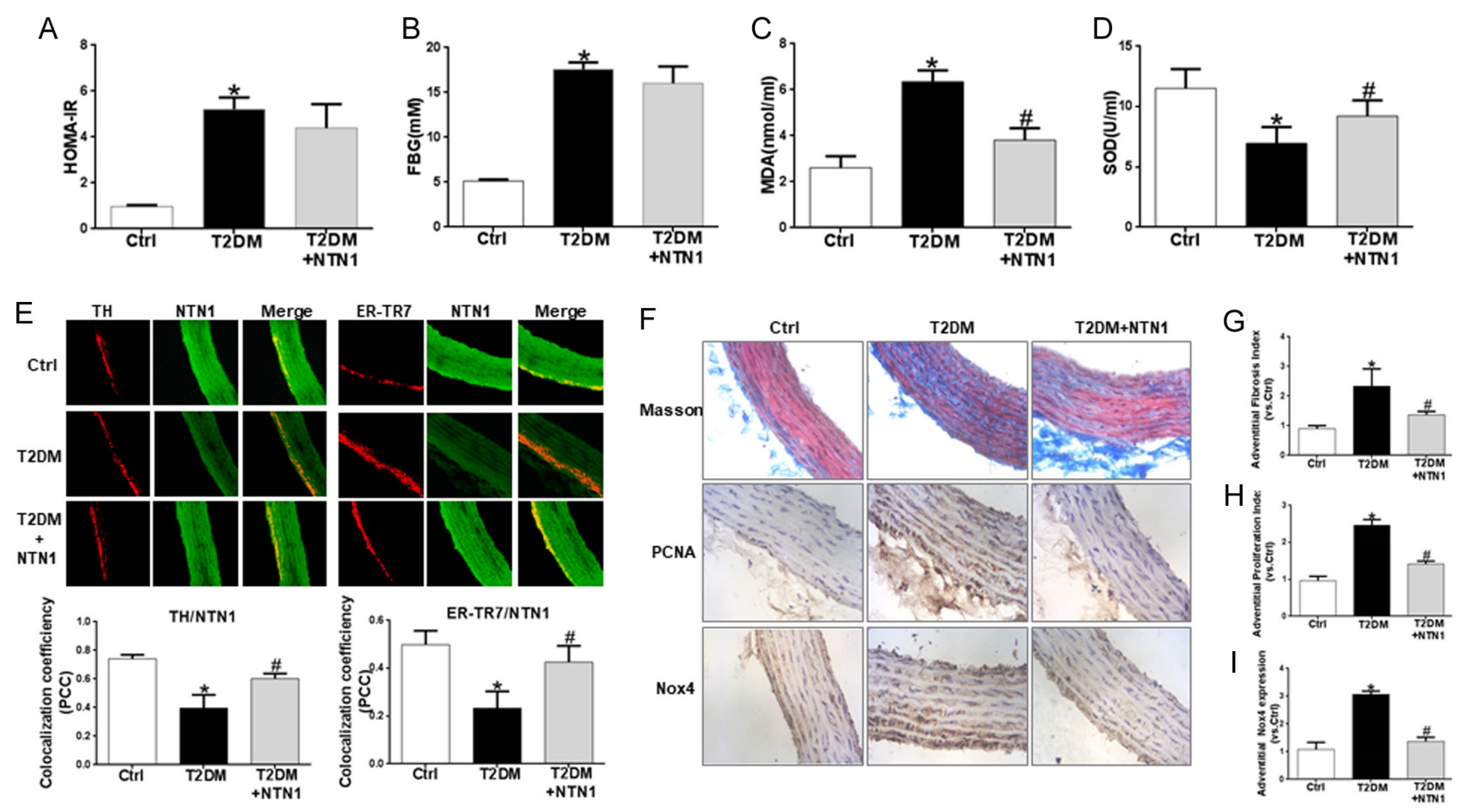

Figure 5

Netrin-1 attenuated vascular adventitia remodeling in T2DM rats. Male SD rats were fed HFD and a low dose of STZ (40 mg/kg, i.p.) to develop a rat model of T2DM that were treated with recombinant netrin-1 (120 $\mu \mathrm{g} / \mathrm{kg} /$ per $48 \mathrm{~h}$, intraperitoneally) for 6 weeks. Summarized data showed the HOMA-IR, FBG, MDA, and SOD, respectively in the serum (A, B, C, and D). Data are means \pm S.E.M., $n=6$ rats. Representative immunofluorescence staining images and summarized co-efficiency showed the co-localization of netin-1 (green) and TH (red) or ER-TR7 (red) in the aorta (E). Representative Masson staining showed collagen deposition and representative immunohistochemistry images showed the expression of PCNA and Nox4 in the aorta (F). Summarized data showed the adventitial fibrosis index, the adventitial proliferation index and the adventitial Nox4 expression (G, H, and I). Data are means \pm S.E.M., $n=3$ rats. ${ }^{*} P<0.05$ vs Ctrl, $\# P<0.05$ vs T2DM.

remodeling in the T2DM group. Importantly, netrin-1 intervention ameliorated such adventitia thickening.

Compared with the control, the expression of netrin-1 was drastically reduced in the sympathetic neurons and vascular adventitia of T2DM rats (Fig. 5E). Relative to control, ER-TR7 labeling immunofluorescence became stronger in T2DM rats, suggesting that T2DM induced the proliferation of AFs in the aorta. Netrin-1 intervention upregulated netrin-1 expression in the sympathetic neurons and vascular adventitia, accompanied with the inhibition of proliferation of AFs. The co-localization of ER-TR7 and PCNA was used to evaluate the adventitia fibroblasts proliferation. Moreover, the vascular adventitia was isolated from the vessel to detect the protein expression of PCNA. As shown in Supplementary Fig. $6 \mathrm{E}$ and $\mathrm{F}$, compared with the control, the expression of PCNA was drastically elevated in the vascular adventitia of T2DM rats. Compared with the Ctrl group, the UNC5b expression was decreased in the adventitia in T2DM rats, which was upregulated by intervention with netrin-1 (Supplementary Fig. 6G).
In T2DM group, Masson staining showed an increase in collagen deposition in the aorta adventitia, which was relieved by intervention with netrin-1 (Fig. 5F and G). The immunohistochemical analysis revealed that the expression of PCNA and Nox4, as indicated by conspicuous brown granules, were obviously observed in the aorta adventitia of T2DM rats compared to the control group (Fig. 5F). The intensified precipitation of PCNA and Nox4 proteins was dramatically diminished following interventions with netrin-1 (Fig. $5 \mathrm{H}$ and I). Combing with the data in vivo, netrin-1 was one of the key paracrine signaling responsible for the regulation of sympathetic neurons on AFs. Netrin-1 effectively ameliorated vascular adventitial remodeling, which was associated with inhibiting Nox4 expression in AFs.

\section{Discussion}

The current study for the first time demonstrated netrin-1 as a key modulator from SCG neurons to regulate AFs 
function under physiological condition. In T2DM, the paracrine signaling from sympathetic neurons contributed to vascular adventitial remodeling, which was ameliorated by netrin-1 treatment through the UNC5b/cAMP/PKA/Nox4 pathway in AFs.

Sympathetic nerves innervate major arteries and balance the functions of vessel through the trophic factors which released from the varicosities in sympathetic nerve (Zhang \& Faber 2001, Sheng \& Zhu 2018). Netrin-1, as a common signaling molecule between the nervous system and the vascular system, may play a critical role between perivascular sympathetic and vascular adventitia (Carmeliet \& Tessier-Lavigne 2005). Our data found that netrin-1 deficiency in the supernatant from SCG neurons directly stimulated AFs remodeling. Thus, netrin-1 was one of key mediators responsible for this connection between SCG neurons and AFs under physiological condition. Under hyperglycemia environment, the level of netrin-1 in the supernatant from SCG neurons decreased to $100 \mathrm{pg} / \mathrm{mL}$. However, at least $100 \mathrm{ng} / \mathrm{mL}$ could alleviate $\mathrm{CM}(\mathrm{HG})$-induced AFs remodeling. Considering the complexity of trophic factors from SCG neurons, only excessive netrin-1 neutralized the effects from other pathological factors from $\mathrm{CM}(\mathrm{HG})$. All these results together indicated netrin-1 as a crucial modulator from SCG neurons to regulate AFs function. It was also reported that netrin-1 from vascular smooth muscle cells bounded to DCC receptor on sympathetic growth cone (Brunet et al. 2014). Thus, the link between vascular cells and sympathetic cells was further strengthened by the identification of netrin-1 signaling in vascular wall.

The current PCR data found that UNC5b receptor was highly expressed among the specific receptors of netrin-1. UNC5b gene slicing eliminated the benefit of exogenous netrin-1 on vascular remodeling. Thus, UNC5b as a specific receptor mediated the modulation of exogenous netrin-1 on AFs. UNC5b was reported to be a novel inhibitor of $\mathrm{G}_{\mathrm{ia} 2}$ via sequestering the $\alpha$ subunit, thereby increasing intracellular cAMP levels, and such an effect was enhanced by the binding of netrin-1 (Komatsuzaki et al. 2002). PKA, the second messenger on cell growth and differentiation, is the traditional effector of cAMP (Stork $\&$ Schmitt 2002). We demonstrated that netrin-1 bound to UNC5b and then did activate the cAMP/PKA pathway, and activation of the latter attenuated vascular remodeling in AFs. Similarly, cAMP/PKA pathway has been reported as a central determinant of netrin-1 to guide cone growth (Ming et al. 1997). In addition, netrin-1 directly increased cAMP production in dissociated xenopus retinal neurons (Höpker et al. 1999). Thus, cAMP/PKA pathway was regarded as a key regulator of netrin-1 signal transduction in AFs remodeling.

Vascular adventitial remodeling was strongly associated with oxidative stress (Haurani \& Pagano 2007). Exogenous netrin-1 indeed suppressed NADPH oxidasederived ROS production in $\mathrm{CM}(\mathrm{HG})$-incubated AFs, suggesting an anti-oxidative property of netrin-1. Nox4 is the most predominant and abundant NADPH oxidase isoforms in AFs (Haurani \& Pagano 2007). We found that exogenous netrin-1 attenuated the expression of Nox4 and p22phox as well as its co-localization with each other. Thus, Nox4 was the main source of ROS responsible for the regulation of exogenous netrin-1 on vascular adventitial remodeling. $\mathrm{H}_{2} \mathrm{O}_{2}$, rather than $\mathrm{O}_{2} \cdot{ }^{-}$, has been suggested as the major production of ROS via Nox4 (Gorin $\&$ Block 2013). Here, both intra- and extracellular $\mathrm{H}_{2} \mathrm{O}_{2}$ caused by $\mathrm{CM}(\mathrm{HG})$ was implicated in netrin-1/UNC5b/ cAMP/PKA/Nox4 pathway. Moreover, $\mathrm{H}_{2} \mathrm{O}_{2}$ indeed induced the proliferation, collagen synthesis, and cell migration in AFs.

T2DM rats produced by HFD/low STZ provided a wellcharacterized model of vascular adventitia remodeling, which was verified by HE staining, Masson staining, and the immunohistochemical analysis in vascular adventitia. Netrin-1 inhibited collagen deposition and fibroblasts proliferation, which was consistent with influence of netrin-1 in AFs in vitro. Reduced SOD activity and increased MDA level reflected a condition of oxidative stress in T2DM rats, which was associated with local Nox4 activation in vascular adventitia. The level of oxidative stress in rat serum was normalized by the intervention of netrin-1 in vivo.

In the current study, immunofluorescence staining of aortic rings supported the notion that sympathetic nerve fibers were indeed confined to the adventitial side of the media muscle coat and surrounded the periphery of the rat aorta. Further, the co-localization of netrin-1 and TH or ER-TR7 suggested that netrin-1 existed in the perivascular sympathetic neurons and vascular adventitia. Diabetes resulted in a significant decrease of netrin-1 in the aorta. Additionally, the expression of ER-TR7 increased in the aorta of the T2DM group indicated fibroblasts proliferation and adventitial thickening. However, there was no significant change in TH expression in the aorta in T2DM rats, possibly because TH expression kept constant even under hyperglycemia (Li \& Grimes 1993, Xuan et al. 2015). Besides the pathological changes in vascular adventitia, the injury on the media and endothelium was also observed, which might be alleviated by netrin-1 in T2DM rats. 
In conclusion, we demonstrated that netrin- 1 is a key modulator which linked SCG neurons and AFs. In T2DM, the paracrine signaling from SCG neurons caused the exacerbation of vascular adventitial remodeling, which was ameliorated by netrin- 1 treatment through the UNC5b/cAMP/PKA/Nox4 pathway. These findings suggested that netrin-1 plays a key role in the homeostasis between the perivascular sympathetic neurons and vascular adventitia. Thus, netrin-1 is a promising therapeutic agent in alleviating vascular adventitial remodeling in T2DM.

\section{Supplementary materials}

This is linked to the online version of the paper at https://doi.org/10.1530/ JOE-19-0258.

\section{Declaration of interest}

The authors declare that there is no conflict of interest that could be perceived as prejudicing the impartiality of the research reported.

\section{Funding}

This work was supported by the National Natural Science Foundation of China (No. 81570413, No. 81773732, and No. 81973509) and 'Double FirstClass' University project (No. CPU2018GY11).

\section{References}

Barman SA, Chen F, Su Y, Dimitropoulou C, Wang Y, Catravas JD, Han W, Orfi L, Szantai-Kis C, Keri G, et al. 2014 NADPH oxidase 4 is expressed in pulmonary artery adventitia and contributes to hypertensive vascular remodeling. Arteriosclerosis, Thrombosis, and Vascular Biology 34 1704-1715. (https://doi.org/10.1161/ ATVBAHA.114.303848)

Brunet I, Gordon E, Han J, Cristofaro B, Broqueres-You D, Liu C, Bouvrée K, Zhang J, del Toro R, Mathivet T, et al. 2014 Netrin-1 controls sympathetic arterial innervation. Journal of Clinical Investigation 124 3230-3240. (https://doi.org/10.1172/JCI75181)

Burnstock G 2008 Non-synaptic transmission at autonomic neuroeffector junctions. Neurochemistry International 52 14-25. (https://doi. org/10.1016/j.neuint.2007.03.007)

Campanucci V, Krishnaswamy A \& Cooper E 2010 Diabetes depresses synaptic transmission in sympathetic ganglia by inactivating nAChRs through a conserved intracellular cysteine residue. Neuron $6 \mathbf{6}$ 827-834. (https://doi.org/10.1016/j.neuron.2010.06.010)

Carmeliet P \& Tessier-Lavigne M 2005 Common mechanisms of nerve and blood vessel wiring. Nature 436 193-200. (https://doi. org/10.1038/nature03875)

Chen J, Lai J, Yang L, Ruan G, Chaugai S, Ning Q, Chen C \& Wang DW 2016 Trimetazidine prevents macrophage-mediated septic myocardial dysfunction via activation of the histone deacetylase sirtuin 1. British Journal of Pharmacology 173 545-561. (https://doi.org/10.1111/ bph.13386)

Cheng X, Ma Y, Moore M, Hemmings BA \& Taylor SS 1998 Phosphorylation and activation of cAMP-dependent protein kinase

(C) 2020 Society for Endocrinology Published by Bioscientifica Ltd. Printed in Great Britain by phosphoinositide-dependent protein kinase. PNAS 95 9849-9854. (https://doi.org/10.1073/pnas.95.17.9849)

Damon DH 2000 VSM growth is stimulated in sympathetic neuron/VSM cocultures: role of TGF-beta2 and endothelin. American Journal of Physiology: Heart and Circulatory Physiology 278 H404-H411. (https:// doi.org/10.1152/ajpheart.2000.278.2.H404)

Deiana L, Carru C, Pes G \& Tadolini B 1999 Spectrophotometric measurement of hydroperoxides at increased sensitivity by oxidation of Fe2+ in the presence of xylenol orange. Free Radical Research $\mathbf{3 1}$ 237-244. (https://doi.org/10.1080/10715769900300801)

Delloye-Bourgeois C, Fitamant J, Paradisi A, Cappellen D, Douc-Rasy S, Raquin MA, Stupack D, Nakagawara A, Rousseau R, Combaret V, et al. 2009 Netrin-1 acts as a survival factor for aggressive neuroblastoma. Journal of Experimental Medicine 206 833-847. (https://doi. org/10.1084/jem.20082299)

Fan Y, Shen F, Chen Y, Hao Q, Liu W, Su H, Young WL \& Yang G 2008 Overexpression of netrin-1 induces neovascularization in the adult mouse brain. Journal of Cerebral Blood Flow and Metabolism $\mathbf{2 8}$ 1543-1551. (https://doi.org/10.1038/jcbfm.2008.39)

Gorin Y \& Block K 2013 Nox4 and diabetic nephropathy: with a friend like this, who needs enemies? Free Radical Biology and Medicine $\mathbf{6 1}$ 130-142. (https://doi.org/10.1016/j.freeradbiomed.2013.03.014)

Haurani MJ \& Pagano PJ 2007 Adventitial fibroblast reactive oxygen species as autacrine and paracrine mediators of remodeling: bellwether for vascular disease? Cardiovascular Research 75 679-689. (https://doi.org/10.1016/j.cardiores.2007.06.016)

Höpker VH, Shewan D, Tessier-Lavigne M, Poo M \& Holt C 1999 Growthcone attraction to netrin-1 is converted to repulsion by laminin-1. Nature 401 69-73. (https://doi.org/10.1038/43441)

Kacem K \& Sercombe R 2006 Differing influence of sympathectomy on smooth muscle cells and fibroblasts in cerebral and peripheral muscular arteries. Autonomic Neuroscience: Basic and Clinical 124 38-48. (https://doi.org/10.1016/j.autneu.2005.11.003)

Kacem K \& Sercombe R 2008 Similar pathological effects of sympathectomy and hypercholesterolemia on arterial smooth muscle cells and fibroblasts. Acta Histochemica 110 302-313. (https://doi. org/10.1016/j.acthis.2007.11.007)

Kennedy TE, Serafini T, de la Torre JR \& Tessier-Lavigne M 1994 Netrins are diffusible chemotropic factors for commissural axons in the embryonic spinal cord. Cell 78 425-435. (https://doi. org/10.1016/0092-8674(94)90421-9)

Komatsuzaki K, Dalvin S \& Kinane TB 2002 Modulation of G i 2 signaling by the axonal guidance molecule UNC5H2. Biochemical and Biophysical Research Communications 297 898-905. (https://doi org/10.1016/s0006-291x(02)02277-5)

Kung ML, Hsieh SL, Wu CC, Chu TH, Lin YC, Yeh BW \& Hsieh S 2015 Enhanced reactive oxygen species overexpression by $\mathrm{CuO}$ nanoparticles in poorly differentiated hepatocellular carcinoma cells Nanoscale 7 1820-1829. (https://doi.org/10.1039/c4nr05843g)

Layne K, Ferro A \& Passacquale G 2015 Netrin-1 as a novel therapeutic target in cardiovascular disease: to activate or inhibit? Cardiovascular Research 107 410-419. (https://doi.org/10.1093/cvr/cvv201)

Li H \& Grimes P 1993 Adrenergic innervation of the choroid and iris in diabetic rats. Current Eye Research 12 89-94. (https://doi org/10.3109/02713689308999500)

Li Q, Su J, Jin SJ, Wei W, Cong XD, Li XX \& Xu M 2018 Argirein alleviates vascular endothelial insulin resistance through suppressing the activation of Nox4-dependent $\mathrm{O} 2$ - production in diabetic rats. Free Radical Biology and Medicine 121 169-179. (https://doi.org/10.1016/j. freeradbiomed.2018.04.573)

Li X, Wang HF, Li XX \& Xu M 2019 Contribution of acid sphingomyelinase to angiotensin II-induced vascular adventitial remodeling via membrane rafts/Nox2 signal pathway. Life Sciences 219 303-310. (https://doi.org/10.1016/j.lfs.2019.01.028)

Madison RD, Zomorodi A \& Robinson GA 2000 Netrin-1 and peripheral nerve regeneration in the adult rat. Experimental Neurology 161 563-570. (https://doi.org/10.1006/exnr.1999.7292) 
Michel JB, Thaunat O, Houard X, Meilhac O, Caligiuri G \& Nicoletti A 2007 Topological determinants and consequences of adventitial responses to arterial wall injury. Arteriosclerosis, Thrombosis, and Vascular Biology 27 1259-1268. (https://doi.org/10.1161/ATVBAHA.106.137851)

Ming GL, Song HJ, Berninger B, Holt CE, Tessier-Lavigne M \& Poo MM 1997 cAMP-dependent growth cone guidance by Netrin-1. Neuron 19 1225-1235. (https://doi.org/10.1016/s0896-6273(00)80414-6)

Mohamed R, Jayakumar C, Ranganathan PV, Ganapathy V \& Ramesh G 2012 Kidney proximal tubular epithelial-specific overexpression of netrin-1 suppresses inflammation and albuminuria through suppression of COX-2-mediated PGE2 production in streptozotocininduced diabetic mice. American Journal of Pathology 181 1991-2002. (https://doi.org/10.1016/j.ajpath.2012.08.014)

Passacquale G, Phinikaridou A, Warboys C, Cooper M, Lavin B, Alfieri A, Andia ME, Botnar RM \& Ferro A 2015 Aspirin-induced histone acetylation in endothelial cells enhances synthesis of the secreted isoform of netrin-1 thus inhibiting monocyte vascular infiltration. British Journal of Pharmacology 172 3548-3564. (https://doi. org/10.1111/bph.13144)

Prior KK, Leisegang MS, Josipovic I, Lowe O, Shah AM, Weissmann N, Schroder K \& Brandes RP 2016 CRISPR/Cas9-mediated knockout of p22phox leads to loss of Nox1 and Nox4, but not Nox5 activity. Redox Biology 9 287-295. (https://doi.org/10.1016/j.redox.2016.08.013)

Rajasekharan S \& Kennedy TE 2009 The netrin protein family. Genome Biology 10 239. (https://doi.org/10.1186/gb-2009-10-9-239)

Ranganathan P, Mohamed R, Jayakumar C, Brands MW \& Ramesh G 2015 Deletion of UNC5B in kidney epithelium exacerbates diabetic nephropathy in mice. American Journal of Nephrology 41 220-230. (https://doi.org/10.1159/000381428)

Salum E, Kampus P, Zilmer M, Eha J, Butlin M, Avolio AP, Põdramägi T, Arend A, Aunapuu M \& Kals J 2012 Effect of vitamin D on aortic remodeling in streptozotocin-induced diabetes. Cardiovascular Diabetology 11 58-58. (https://doi.org/10.1186/1475-2840-11-58)

Sheng Y \& Zhu L 2018 The crosstalk between autonomic nervous system and blood vessels. International Journal of Physiology, Pathophysiology and Pharmacology 10 17-28.

Soares-da-Silva P \& Azevedo I 1985 Fibroblasts and sympathetic innervation of blood vessels. Journal of Vascular Research 22 278-285. (https://doi.org/10.1159/000158614)
Stenmark KR, Yeager ME, El Kasmi KC, Nozik-Grayck E, Gerasimovskaya EV, Li M, Riddle SR \& Frid MG 2013 The adventitia: essential regulator of vascular wall structure and function. Annual Review of Physiology 75 23-47. (https://doi.org/10.1146/annurevphysiol-030212-183802)

Stork PJS \& Schmitt JM 2002 Crosstalk between cAMP and MAP kinase signaling in the regulation of cell proliferation. Trends in Cell Biology 12 258-266. (https://doi.org/10.1016/s0962-8924(02)02294-8)

Takac I, Schröder K, Zhang L, Lardy B, Anilkumar N, Lambeth JD, Shah AM, Morel F \& Brandes RP 2011 The E-loop is involved in hydrogen peroxide formation by the NADPH oxidase Nox4. Journal of Biological Chemistry 286 13304-13313. (https://doi.org/10.1074/jbc. M110.192138)

Toque HA, Fernandez-Flores A, Mohamed R, Caldwell RB, Ramesh G \& Caldwell RW 2017 Netrin-1 is a novel regulator of vascular endothelial function in diabetes. PLOS ONE 12 e0186734. (https://doi. org/10.1371/journal.pone.0186734)

Vandesompele J, De Preter K, Pattyn F, Poppe B, Van Roy N, De Paepe A $\&$ Speleman F 2002 Accurate normalization of real-time quantitative RT-PCR data by geometric averaging of multiple internal control genes. Genome Biology 3 RESEARCH0034. (https://doi.org/10.1186/ gb-2002-3-7-research0034)

Wei W, Li XX \& Xu M 2019 Inhibition of vascular neointima hyperplasia by FGF21 associated with FGFR1/Syk/NLRP3 inflammasome pathway in diabetic mice. Atherosclerosis 289 132-142. (https://doi. org/10.1016/j.atherosclerosis.2019.08.017)

Xing Y, Lai J, Liu X, Zhang N, Ming J, Liu H \& Zhang X 2017 Netrin-1 restores cell injury and impaired-angiogenesis in vascular endothelial cells. Journal of Molecular Endocrinology 58 167-177. (https://doi. org/10.1530/JME-16-0239)

Xuan YL, Wang Y, Xue M, Hu HS, Cheng WJ, Li XR, Yin J, Yang N \& Yan SH 2015 In rats the duration of diabetes influences its impact on cardiac autonomic innervations and electrophysiology. Autonomic Neuroscience: Basic and Clinical 189 31-36. (https://doi.org/10.1016/j. autneu.2015.01.003)

Zhang H \& Faber JE 2001 Trophic effect of norepinephrine on arterial intima-media and adventitia is augmented by injury and mediated by different alpha1-adrenoceptor subtypes. Circulation Research 89 815-822. (https://doi.org/10.1161/hh2101.098379)

Received in final form 22 November 2019

Accepted 16 December 2019

Accepted Manuscript published online 17 December 2019 https://joe.bioscientifica.com https://doi.org/10.1530/JOE-19-0258 (c) 2020 Society for Endocrinology Published by Bioscientifica Ltd. Printed in Great Britain 